\title{
Modification of Glauber theory for dynamic scattering of electrons by polyatomic molecules*
}

\author{
L. S. Bartell \\ Department of Chemistry, The University of Michigan, Ann Arbor, Michigan 48104 \\ (Received 7 July 1975) \\ In its original form, Glauber scattering theory achieves a considerable simplification by neglecting several \\ factors of modest consequence in atomic scattering but of importance in molecular scattering. These \\ include, in particular, the spread of the shadow cast by a scatterer as a wave propagates downstream in the \\ target. Although such effects do not matter in the original range of validity of the theory, the characteristic \\ distances in molecules restrict the unmodified theory to an excessively small angular range. The present \\ treatment introduces phase factors to take into account the distribution of scatterers in the incident \\ direction and the propagation of the scattered waves in the target. This appreciably extends the formal \\ range of applicability.
}

\section{INTRODUCTION}

Electron diffraction has become an increasingly powerful tool in studies of structures and conformations of molecules. Indeed, the accuracy with which intensity measurements can now be made exceeds that of the dif fraction theory used in interpreting the measurements in certain cases, most notably those in which heavy at oms are present. A resolution of the persistent discrepancies between vapor -phase experiment and applied theory has been sought in "intramolecular multiple scattering" or "dynamic scattering," and a number of theoret ical treatments have appeared in recent years. ${ }^{1-10}$ These have been tested mainly by comparison with the data for a single example, ${ }^{11} \operatorname{ReF}_{6}$, where all treatments gave rather similar results. The agreement between theory and experiment was encouraging for this particularly simple molecule in which bond angles are all either $90^{\circ}$ or $180^{\circ}$ and in the similar case of $\mathrm{XeOF}_{4}{ }^{12,13}$ Poorer results were obtained in unpublished studies ${ }^{14}$ of $\operatorname{ReF}_{7}$ and $\mathrm{IF}_{7}$, and an application to the problem of dynamic scattering corrections for very small, randomly oriented crystallites revealed serious deficiencies. ${ }^{15}$ The reasons for these deficiencies are discussed in the following.

The first treatments ${ }^{1-6}$ of multiple scattering made use of the second Born approximation and involved dif ficult numerical integrations or analytical integrations of expansions in which truncation errors of uncertain magnitude were introduced. The more recent treatments ${ }^{7-10}$ have exploited Glauber's approach ${ }^{16}$ to high energy scattering. The simplicity and transparency of model in this eikonal theory are great virtues in reducing to tractability the six-dimensional integrals encountered in the general theory in the orientational averaging of products of three-dimensional integrals representing scattered amplitudes. The preliminary successes, unfortunately, masked the fact that the eikonal model was applied beyond its range of validity. It is the purpose of the present article to show how to extend the range of validity of theoretical expressions at a trivial increase in complexity.

\section{PHYSICAL INTERPRETATION}

Before introducing a mathematical treatment, it is helpful to present the physical interpretation of the cor- rections required for the standard kinematic treatment of the elastic scattering of electrons. In kinematic theory, it is considered that a plane wave passes through the target, undistorted in phase by previous encounters with atoms, unattenuated by previous scatterings, and unaugmented by scatterings from other regions of the target. Electrons are not conserved. In a more realistic treatment of small systems, an important consider ation is that each atom encountered by the incident wave casts a shadow. The depletion of flux downstream of the encounter in the forward direction exactly balances the flux scattered away from the incident direction of propagation. Subsequent scatterings by downstream atoms are more feeble than scatterings given by kinematic theory, and, hence, the kinematic intensity corresponding to scatterings from nearly eclipsed atoms must be corrected downwards, to conserve energy. If the target is more than a few atoms deep, the extra (scattered) intensity arriving at certain sites from directions other than the original incident direction must also be taken into account. In the present cases of small molecules and very small crystallites, however, this complication can be neglected.

Glauber theory in its simplest variant conserves energy by the simple expedient of not allowing the incident electron trajectories to be diverted from their original directions by scattering until the electrons emerge from the target. A semiclassical phase shift of electrons induced by the passage of the beam through the atomic potentials is computed, however, and this distorts the wave front emerging from the target. After emerging, the wave front is allowed to propagate properly from tar get to detector. In effect, the target potential has been projected into a thin film perpendicular to the incident beam. ${ }^{17}$ The advantage of this approach is that it simplifies a difficult integral over target volume to a twodimensional integral over the emerging wave front. Results are quite good within the fundamental limit of validity for scattering angle, namely, ${ }^{16}$

$$
\theta^{2} \ll 1 / k d,
$$

or

$$
s^{2} \ll k / d,
$$

where $d$ is the distance over which the potential energy varies and $s=|\mathbf{s}|$, with $\mathbf{s}=\mathbf{k}_{\text {inc }}-\mathbf{k}_{\text {scatt }}$. 
Since atomic potentials responsible for scattering to large $s$ are only a few hundredths of an angstrom unit across, Glauber theory gives a satisfactory representation of the scattering by single atoms out to fairly large $s$. Its limitations in the case of molecules are twofold. First, interatomic distances ranging from 2 to $10 \AA$, say, would restrict $s$ for $40 \mathrm{kV}$ electrons $\left(k \approx 100 \AA^{-1}\right)$ to values much less than $3 \AA^{-1}$, according to Eq. (1b), whereas experimental studies commonly go out to $30-$ $60 \AA^{-1}$. Atoms that are eclipsed (the important configuration in multiple scattering corrections) give intensity interference features of the form $\cos \left(\mathbf{s} \cdot \mathrm{K}_{0} R\right)$, where $\mathrm{K}_{0}$ is the unit vector in the incident direction and $R$ is the atomic separation. These features are absent in the variant of Glauber theory we modify in the following. Secondly, the shadow cast by an atom spreads as it propagates downstream. This has the following crucial ef fect on the multiple scattering corrections:

Consider the group of three atoms, $i, j$, and $k$ illus trated in Fig. 1. The most important dynamic correction term for polyatomic molecules is of the type corresponding to a wave scattered by atom $j$ interfering with a wave scattered by atom $k$ when atom $j$ is in the shadow of atom $i$. The associated interference pattern, aver aged over all orientations of atom $k$ about the $i j$ axis, is a zeroth-order Bessel function, $J_{0}\left(s r_{k}\right)$, the pattern characteristic of an annular slit with a radius $\boldsymbol{r}_{\boldsymbol{k}}$. This particular ( $i$ eclipsing $j$ ) contribution to the total orientational average is incorrectly calculated by the kinematic theory because kinematic theory neglects any attenuation or phase shift induced in the wave incident upon $j$ by passage through $i$. Dynamic corrections simply alter the coefficient of the $J_{0}\left(s r_{k}\right)$ term to compensate for the neglect. The $J_{0}\left(s r_{k}\right)$ component is damped (as a function of $s$ ) by the delocalization of the projected $k$ to $j$ distance when intensities are averaged over orientations including those in which $j$ is not exactly eclipsed by atom $i$. Standard Glauber theory in Ref. 9 handles correctly that part of the allowed deviation from perfect eclipsing which arises from the natural breadths of the atoms. Atoms partially overlap even when their nuclei are not exactly eclipsed. A significant source of modulation of $J_{0}\left(s r_{k}\right)$ is left out by Glauber theory, however, owing to the fact that no true shadow is allowed to be cast by atom $i$ because the phase-shifted wave is not allowed to spread as it propagates from $i$ to $j$. The spread of a bona fide shadow in-

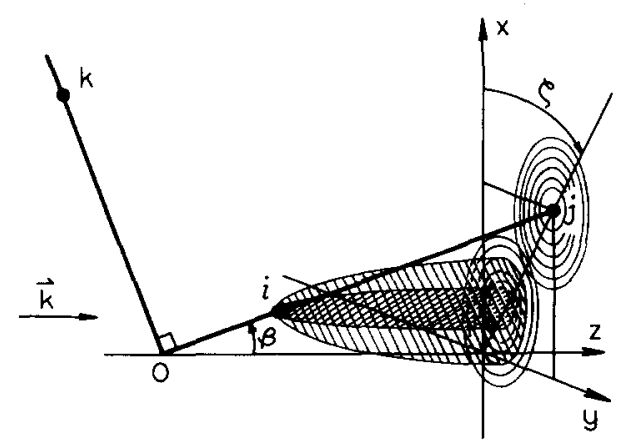

FIG. 1. Three-atom group $i \rightarrow j, k$ illuminated from the left by a plane wave. Atom $j$ lies in the shadow cast by atom $i$ when angle $\beta$ is small. Origin $o$ is chosen to make angle $k-o-i j=90^{\circ}$. creases the range of deviation from perfect eclipsing of $j$ by $i$ over which the three-atom correction is significant. For some structures this results in a much faster attenuation of the three-atom $J_{0}\left(s r_{k}\right)$ interference ripples than given by Glauber theory.

\section{GLAUBER SCATTERING BY POLYATOMIC MOLECULES}

In order to construct the new model for polyatomic molecules and compare it with various previous treatments, it is necessary to outline essential features of Glauber scattering theory. The Glauber scattering factor for a plane wave $\exp (i \mathbf{k} \cdot \mathbf{r})$ encountering a system of atoms is ${ }^{16}$

$$
\begin{aligned}
f\left(\mathbf{k}^{\prime}, \mathbf{k}\right)= & -\frac{2 m}{4 \pi \hbar^{2}} \int d^{2} p d z \exp \left[i\left(\mathbf{k}-\mathbf{k}^{\prime}\right) \cdot(\mathbf{p}+\mathbf{K} z)\right] \\
& \times V(\mathbf{p}+\mathbf{K} z) \exp [i \Xi(p, z)],
\end{aligned}
$$

where the semiclassical phase shift $\Xi$ is given by

$$
\Xi(\mathrm{p}, z)=-(\hbar v)^{-1} \int_{-\infty}^{z} d z^{\prime} V\left(\mathrm{p}+\mathrm{K} z^{\prime}\right) \text {. }
$$

In the simplest variant (SG) of Glauber theory the unit vector $K=K_{0}$ is chosen to lie in the direction of the incident momentum $\mathbf{k}$ and the small quantity $\left(\mathbf{k}-\mathbf{k}^{\prime}\right) \cdot \mathrm{K}_{0} z$ in the first exponential function of Eq. (2) is disregarded. A modified variant (MG), representing an improvement in some respects, is to choose $K$ to lie in the average direction $\mathbf{k}+\mathbf{k}^{\prime}$ of incident and scattered direction. This rigorously makes $\left(\mathbf{k}-\mathbf{k}^{\prime}\right) \cdot \mathbf{K} z$ vanish. In either approach the impact parameter $\mathrm{p}$ is perpendicular to, and $z$ is taken along, the chosen $K$ vector. In either case, Eq. (2) reduces to

$$
f\left(s^{\prime}\right)=-\frac{i k}{2 \pi} \int d^{2} p \exp \left(i s^{\prime} \cdot \mathbf{p}\right)\{\exp [i \chi(\mathbf{p})]-1\},
$$

where $\chi(p)$ is $\Xi(\mathbf{p}, z)$ of Eq. (2) evaluated at $z=\infty$ and, in MG theory, $\mathbf{s}^{\prime}=\mathbf{s}=\mathbf{k}-\mathbf{k}^{\prime}$, while in SG theory, $\mathbf{s}^{\prime}$ with magnitude $k \theta$ is represented adequately by $\mathbf{s}^{\prime} \approx \mathbf{k}-\mathbf{k}^{\prime}=\mathbf{s}$ over the range of validity of the theory. A given pair of atoms $i$ and $j$ with $V=V_{i}+V_{1}$ and, hence, $\chi=\chi_{i}+\chi_{j}$, produces single and double scattering amplitudes in accord with the identity

$$
\begin{aligned}
{[\exp (i \chi)-1]=} & \exp \left[i\left(\chi_{i}+\chi_{j}\right)\right]-1 \\
= & {\left[\exp \left(i \chi_{i}\right)-1\right]+\left[\exp \left(i \chi_{j}\right)-1\right] } \\
& +\left[\exp \left(i \chi_{i}\right)-1\right]\left[\exp \left(i \chi_{j}\right)-1\right] .
\end{aligned}
$$

This result applied to Eq. (4) yields

$$
f(\mathbf{s})=f_{i} \exp \left(i \mathrm{~s} \cdot \mathbf{p}_{i}\right)+f_{j} \exp \left(i \mathbf{s} \cdot \mathbf{p}_{j}\right)+f_{i j},
$$

where the scattering factor $f_{i j}$ stemming from the last rhs term of Eq. (5) represents the nonkinematic contribution. If a third atom $k$ is also present, the singledouble scattering $i-j, k$ averaged in orientation over the hemisphere with $j$ in the lee of $i$, then, is

$$
\langle d \sigma / d \Omega\rangle_{i-j, k}=2 \operatorname{Re}\left\langle f_{k}^{*} \exp \left(-i s \cdot \mathrm{p}_{k}\right) f_{i j}\right\rangle
$$

with the Eulerian angles characterizing the molecular orientation taken over the ranges $0<\alpha<2 \pi, 0<\gamma<2 \pi$, $0<\beta<\pi / 2$. The key to a simple evaluation of Eq. (7) is to pivot the molecular fragment shown in Fig. 1 about 
the special point $o$ in the rotational averaging and to introduce the coordinate transformation $\alpha, \beta, \gamma, \mathbf{p} \rightarrow \mathbf{b}_{i}$, $\mathbf{b}_{f}, \phi$ detailed in Ref. 9 . We adopt the definitions

$$
\begin{array}{lll}
\mathbf{r}_{l}=\mathbf{o l}, & l=i, j, k \\
\mathbf{b}_{l}=\mathbf{p}-\mathbf{p}_{l} & \\
\mathbf{s}_{i}=\left(r_{j} / r_{i j}\right) \mathbf{s}, & r_{j}=\mathbf{K} \cdot \mathbf{r}_{j} & (\text { at } \beta=0) \\
\mathbf{g}_{j}=-\left(r_{i} / r_{i j}\right) \mathbf{s}, & r_{i}=\mathbf{K} \cdot \mathbf{r}_{i} & (\text { at } \beta=0)
\end{array}
$$

and

$$
\left\langle\frac{d \sigma}{d \Omega}\right\rangle_{i \rightarrow j, k}=-2 \operatorname{Re}\left\{\frac{i k}{8 \pi^{2} r_{i j}^{2}} J_{0}\left(s r_{k}\right) f_{k}^{*}(s) \int d^{2} b_{i} \exp \left(i \mathbf{s}_{i} \cdot \mathbf{b}_{i}\right)\left[\exp \left(i \chi_{i}\right)-1\right] \int d^{2} b_{j} \exp \left(i \mathbf{s}_{j} \cdot \mathbf{b}_{j}\right)\left[\exp \left(i \chi_{j}\right)-1\right]\right\}
$$

or, identifying atomic scattering factors by means of Eq. (4), to

$$
\langle d \sigma / d \Omega\rangle_{i-j, k}=-\left(1 / k r_{i j}^{2}\right) J_{0}\left(s r_{k}\right) \operatorname{Im}\left[f_{k}^{*}(s) f_{i}\left(s_{i}\right) f_{j}\left(s_{j}\right)\right] .
$$

The other hemisphere with $k, j \rightarrow i$, receives a corresponding treatment. It is instructive to examine the significance of the arguments $s_{i}$ and $s_{j}$ (rather than $s$ or 0 ) of scattering factors $f_{i}$ and $f_{j}$. The modulation of the Bessel function by $\operatorname{Im}\left[f_{k}^{*}(s) f_{i}\left(s_{i}\right) f_{j}\left(s_{j}\right)\right]$ expresses the influence of the averaging of the $(i \rightarrow j, k)$ term over all orientations of effective eclipsing of $j$ by $i$ accessible within the natural breadths of atoms $i$ and $j$. When atom $j$ is far removed from atom $k$ in the $z$ direction, for example, $s_{i}$ greatly exceeds $s$ and $\left|f\left(s_{i}\right)\right|$ is much smaller than $|f(s)|$. This extra damping action of $\left|f\left(s_{i}\right)\right|$ embodies the greater physical delocalization of the perpendic ular interference distance $\left(r_{k}-z_{j} \sin \beta \sin \zeta\right)$ between atom $k$ and atom $j$ for a given angular delocalization $\beta$ from perfect eclipsing of $i$ and $j$.

\section{SHADOW PROPAGATION MODEL}

Glauber theory applied to a polyatomic fragment $i j k$ corresponds mathematically to (a) condensing $V_{l}(\mathrm{p}, z)$ for atom $l$ into a projected potential

$$
\mathrm{V}_{l}^{\ell}\left(\mathrm{p}, z_{l}\right)=\delta\left(z-z_{l}\right) \int_{-\infty}^{\infty} d z^{\prime} V_{l}\left(\mathrm{p}, z^{\prime}\right)
$$

contained, as it were, in a thin film perpendicular to $K$ and passing through the nucleus of atom $l$, and (b) constraining electron trajectories to be parallel to $\mathrm{K}$ while within the target. In the shadow propagation model, con- dition (a) above is retained but condition (b) is replaced by a less severe one. The wave scattered by atom $i$ is allowed to propagate normally in the interfilm forcefree space between $i$ and $j$ until it encounters the plane through $j$. The shadow amplitude experienced by atom $j$, of course, weights the orientational average $\langle d \sigma / d \Omega\rangle_{i-j, k}$.

If the new model is to make physical sense, the shadow cast by atom $i$ cannot be arbitrarily bent to run with its axis parallel to $\mathbf{k}+\mathbf{k}^{\prime}$ instead of to $\mathbf{k}$. This means that it is more appropriate to patch SG theory than to graft an implausible condition onto MG theory. Fortunately, the patching of SG theory yields a result with proper momentum reversal symmetry while the $\mathrm{MG}$ graft does not. The approach is as follows. Condition (a) above, via Eq. (11), is fed back into Eq. (2). Impact parameters $\mathbf{p}_{l}$ to the various atomic nuclei in Eq. (6) and subsequent equations are replaced by the radial vectors $\mathbf{r}_{l}=\mathbf{p}_{l}+\mathbf{K}_{0} z_{l}$ in order to allow for interference effects arising from scattering centers spaced along $K_{0}$. Although this interference is correctly treated by MG theory, it is absent in SG theory, as mentioned earlier. Because of the above changes, the pair scattering factor $f_{i j}$ acquires a new phase factor $\exp \left(i s \cdot \mathrm{K}_{0} z_{j}\right)$ shown below. The introduction of a proper propagation condition for the wave scattered by atom $i$ requires a replacement of the function $\left\{\exp \left[i \chi_{i}(p)\right]-1\right\}$ with $\chi_{i}$ real by $\left\{\exp \left[i \xi_{i}(p\right.\right.$, $z)]-1\}$ where $\xi_{i}$ is a complex function representing the amplitude shift as well as phase shift for the wavefront as it progresses from atom $i$ to atom $j$. With these changes and the assumption that only small deviations from perfect eclipsing are important, Eq. (9) becomes

$$
\left\langle\frac{d \sigma}{d \Omega}\right\rangle_{i-j, k}=-2 \operatorname{Re}\left\{\frac{i k}{8 \pi^{2} r_{i j}^{2}} J_{0}\left(s r_{k}\right) f_{k}^{*}(s) \exp \left(i \mathbf{s} \cdot \mathbf{K}_{0} z_{j k}\right) \int d^{2} b_{i} \exp \left(i \mathbf{s}_{i} \cdot \mathbf{b}_{i}\right)\left[\exp \left(i \xi_{i}\right)-1\right] \int d^{2} b_{j} \exp \left(i \mathbf{s}_{j} \cdot \mathbf{b}_{j}\right)\left[\exp \left(i \chi_{j}\right)-1\right]\right\},
$$

where $z_{j k}=z_{k}-z_{j}$ for the exactly eclipsed configuration. It can be seen that the coordinate transformation has placed the burden of taking into account the delocalization of $\left(p_{j}-p_{h}\right)$ over the shadow amplitude from atom $i$ entirely into the $b_{i}$ integral of Eq. (12), namely, the scattering factor $f_{i}^{\ell}\left(s_{i}\right)=-\frac{i k}{2 \pi} \int d^{2} b_{i} \exp \left(i{s_{i}}_{i} \cdot \mathrm{b}_{i}\right)\left\{\exp \left[i \xi\left(b_{i}, z_{j}\right)\right]-1\right\}$.

This is the only term whose $s$ dependence is not evident upon inspection. Clearly, $f_{i}^{\xi}$ propagates onward to the detector to become the scattering factor of atom $i$ but 
it includes an interference phase factor that has not yet been determined.

For one extreme limit of approximation $f_{i}^{\xi}\left(s_{i}\right)$ can be evaluated easily. If $V_{\mathbf{i}}(\mathbf{b}, z)$ is a potential of nearly zero range, $\xi(\mathrm{b}, z)$ corresponds to a well-developed spherical wave by the time it reaches atom $j$. If $\gamma_{i j}$ is large compared with the characteristic breadth of $V_{i}$, the wavefunction for an electron passing through atom $i$ is, at $z_{j}$,

$$
\begin{aligned}
\psi(\mathbf{r}) & =\exp \left(i k z_{j}\right) \exp \left(i \xi_{i}\right) \\
& \sim \exp \left(i k z_{j}\right)+f_{i}(\mathbf{s}) r^{-1} \exp (i k r),
\end{aligned}
$$

or, at a point in the vertical plane through atom $j$,

$$
\begin{aligned}
{\left[\exp \left(i \xi_{i}\right)-1\right] } & \sim\left(z_{i j}\right)^{-1} f_{i} \exp \left[i k\left(r-z_{i j}\right)\right] \\
& \approx\left(z_{i j}\right)^{-1} f_{i} \exp \left[\left(i k / 2 z_{i j}\right) b_{i}^{2}\right]
\end{aligned}
$$

by virtue of the pythagorean theorem

$$
r^{2}=z_{i j}^{2}+b_{i}^{2}=r_{i j}^{2}+b_{i}^{2}
$$

for $b_{i} \ll z_{i j}$. Wavefunction (14) extinguishes flux in the forward direction to compensate for laterally scattered radiation. Substitution of Eq. (15) into Eq. (13) yields

$$
f_{i}^{\zeta}\left(s_{i}\right)=f_{i}\left(s_{i}\right) \exp \left[-i\left(r_{i j} / 2 k\right) s_{i}^{2}\right]
$$

and establishes the zero-range limit phase factor $\exp \left[-i\left(r_{i j} / 2 k\right) s_{i}^{2}\right]$. Note also that the other phase factor of Eq. (12) can be written

$$
\exp \left[i \mathrm{~s} \cdot \mathrm{K}_{0} z_{j k}\right]=\exp \left[i\left(r_{i j} / 2 k\right) s_{i} s\right],
$$

in which $s_{i}$ is positive or negative depending upon the sign of $\left(z_{j}-z_{k}\right)$. Therefore, in this limit, the dynamic differential cross section correction $\langle d \sigma / d \Omega\rangle_{i-f_{, k}}$ of Eq. (12) is the same as the Glauber result of Eq. (10) containing

$$
f_{i}\left(s_{i}\right) \equiv\left|f_{i}\left(s_{i}\right)\right| \exp \left[i \eta_{i}\left(s_{i}\right)\right],
$$

except for a phase factor that, in bookkeeping, can be ascribed to atom $i$. Hence, Eq. (10) can be used provided the phase $\eta_{i}\left(s_{i}\right)$ is replaced by an effective phase $H_{i}\left(s_{i}\right)$ given by

$$
H_{i}\left(s_{i}\right)=\eta_{i}\left(s_{i}\right)+\left(r_{i j} / 2 k\right)\left(s s_{i}-s_{i}^{2}\right) .
$$

Presumably, in the other hemisphere with $i$ in the shadow of $j$, the correction $\langle d \sigma / d \Omega\rangle_{k, j \rightarrow i}$ uses an augmented phase for atom $j$ of form

$$
H_{j}\left(s_{j}\right)=\eta_{j}\left(s_{j}\right)+\left(r_{i j} / 2 k\right)\left(s s_{j}-s_{j}^{2}\right) .
$$

Therefore, the shadow propagation model is no more cumbersome to use in routine diffraction analyses than the Glauber model.

Writing the corrections in the above form obscures a more fundamental symmetry. The Schrödinger equation implies a dynamic reversibility such that $f\left(\mathbf{k}^{\prime}, \mathbf{k}\right)$ is identical to $f\left(-\mathbf{k},-\mathbf{k}^{\prime}\right){ }^{16}$ Therefore, if a molecule is reflected across a plane perpendicular to a vector $\mathbf{k}+\mathbf{k}^{\prime}$, the scattering factor is unchanged. It follows that the rotational averaging in the $i-j, k$ hemisphere must be identical to that in the $k, j \rightarrow i$ hemisphere, taking the polar axis along $\mathbf{k}+\mathbf{k}^{\prime}$. Inasmuch as this symmetry holds both for the kinematic and the exact expressions, it should also hold for suitable dynamic corrections and, indeed, it does hold for the intensities corresponding to
Eqs. (19a) and (19b) as well as Eq. (9) and its counterpart. Although MG theory must and does obey this symmetry, SG theory is not expressed in a way to preserve such a symmetry. Nevertheless, the present extension of SG theory should follow the symmetry closely enough to make it a helpful guide. This guide suggests a way to evaluate the "shadow amplitude"

$$
f_{j}^{\xi}\left(s_{j}\right) \equiv f_{j}\left(s_{j}\right) \exp \left(i \delta_{j}\right)
$$

for a scattering potential more general than the zerorange model of Eqs. (14)-(19). Let the quantities pertaining to the $k, j \rightarrow i$ hemisphere be identified by circumflexes and let $V_{i}$ be a zero-range potential. If $\langle d \sigma / d \Omega\rangle_{i-j, k}$ and $\langle d \sigma / d \Omega\rangle_{k, j-i}$ are set equal, it follows that

$$
\begin{aligned}
\operatorname{Im} f_{k}^{*}(s) & f_{i}^{\xi}\left(s_{i}\right) f_{j}\left(s_{j}\right) \exp \left[i \mathbf{s} \cdot \mathbf{K}\left(z_{j}-z_{k}\right)\right] \\
& =\operatorname{Im} f_{k}^{*}(s) f_{i}\left(s_{i}\right) f_{j}^{\xi}\left(s_{j}\right) \exp \left[i \mathbf{s} \cdot \mathbf{K}\left(\tilde{z}_{i}-\tilde{z}_{k}\right)\right],
\end{aligned}
$$

or, because $z_{j}-z_{k}=r_{j}$ and $\tilde{z}_{i}-\tilde{z}_{k}=-r_{i}$,

$$
\begin{aligned}
{\left[\eta_{i}\left(s_{i}\right)\right.} & \left.+\eta_{j}\left(s_{j}\right)-\eta_{k}(s)+\delta_{i}\left(s_{i}\right)+\left(r_{i j} / 2 k\right) s s_{i}\right] \\
& =\left[\eta_{i}\left(s_{i}\right)+\eta_{j}\left(s_{j}\right)-\eta_{k}(s)+\delta_{j}\left(s_{j}\right)+\left(r_{i j} / 2 k\right) s s_{j}\right] .
\end{aligned}
$$

If $-\left(r_{i j} / 2 k\right) s_{i}^{2}$ is substituted for $\delta_{i}\left(s_{i}\right)$ from Eq. (16) and $s_{i}$ is replaced by $\left(s-s_{j}\right)$ according to the definitions of $s_{i}$ and $s_{j}$, it is found that

$$
\delta_{j}\left(s_{j}\right)=-\left(r_{i j} / 2 k\right) s_{j}^{2},
$$

or

$$
f_{j}^{\xi}\left(s_{j}\right)=f_{j}\left(s_{j}\right) \exp \left[-i\left(r_{i j} / 2 k\right) s_{j}^{2}\right]
$$

for a general atom, in the shadow propagation model. This result also secures the proper form for single-scattering, single-scattering interference terms computed from waves emanating from an arbitrary vertical plane displaced from atom $j$.

Taking the above considerations into account, the dynamic correction for the $i j, k$ group over the entire range of orientations can be written

$$
\begin{aligned}
\left\langle\frac{d \sigma}{d \Omega}\right\rangle_{i, k} \approx & -\frac{2}{k r_{i j}^{2}} J_{0}\left(s r_{k}\right)\left|f_{i}\left(s_{i}\right)\right|\left|f_{j}\left(s_{j}\right)\right|\left|f_{k}\left(s_{k}\right)\right| \\
& \times \sin \left[\eta_{i}\left(s_{i}\right)+\eta_{j}\left(s_{j}\right)-\eta_{k}(s)+\left(\frac{r_{i j}}{2 k}\right)\left(s s_{i}-s_{i}^{2}\right)\right] .
\end{aligned}
$$

If molecular vibrations are to be taken into account, the entire dynamic correction of Eq. (23) must be multiplied by the thermal factor $\exp \left(-l_{\perp}^{2} s^{2} / 2\right)$ discussed in Ref. 9 .

Correction terms cubic in $s$ are missing from the argument of the Bessel function of $\mathrm{Eq}$. (23) because of the neglect of terms linear in $\beta$ from the expansion of $(z$, $-z_{k}$ ) and terms quadratic in $\beta$ from the expansion of $\mathbf{p}_{k}(\beta)$ of Eq. (7). Such terms either do not arise or are less important in simple Glauber theory where delocalization over the angle $\beta$ is smaller. In order of magnitude the neglect of these terms restricts Eq. (23) to values of $s$ such that

$$
s^{3} \ll\left(\pi k^{2} r_{i j} / r_{l} r_{k}\right),
$$

where $r_{l}$ is $r_{i}$ or $r_{j}$. So, for $40 \mathrm{kV}$ electrons with $r_{k}$ $\approx r_{i}$, and $r_{i} \sim 2 \AA$, $s$ must be rather less than $26 \AA^{-1}$. 

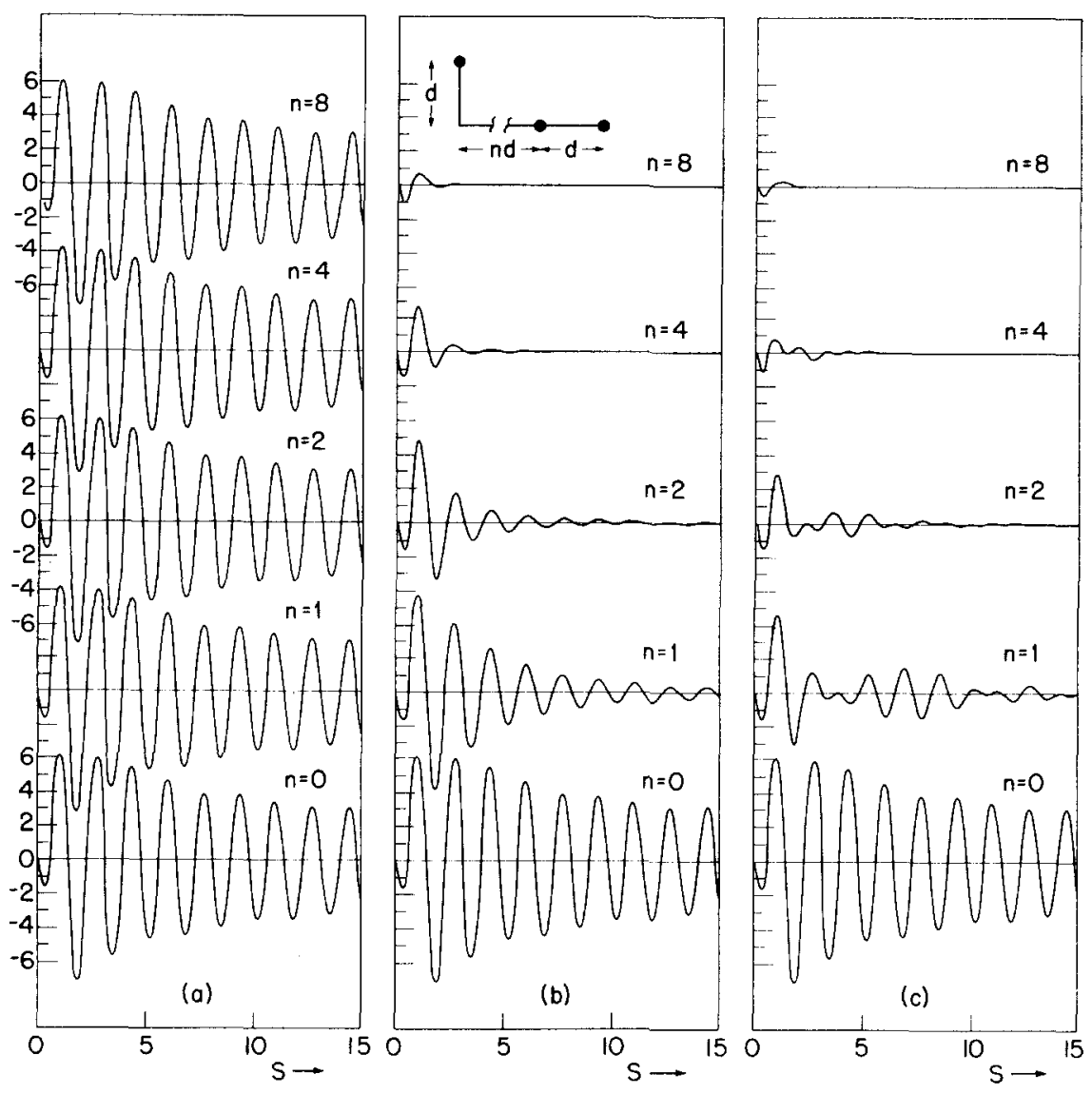

FIG. 2. $10^{5}\langle d \sigma / d s \delta\rangle_{t j, k}$

$\therefore(d \sigma / d \Omega)_{\text {atomic }}$ for threeatom clusters of argon with various configurations depicted over Fig. (b), $d$ $=3.76 \AA$. (a) Theory of Bonham and Peixoto, and Yates. (b) Glauber thoery. (c) Shadow propagation model.
Higher order expansion terms may be treated analytically as outlined in Ref. 9.

\section{DISCUSSION}

It may be asked whether there is anything special about the $\mathrm{ReF}_{6}$ and $\mathrm{XeOF}_{4}$ cases found to be in approximate accord with the Glauber basis result of Eq. (9) and with the Bonham and Peixoto ${ }^{5}$ and Yates ${ }^{8}$ approximations. The answer is that for $90^{\circ} k i j$ bond angles the principal $(i j, k)$ terms in the new equations reduce to the previous Glauber result. ${ }^{9}$ Moreover, for $90^{\circ}$ the product $\left[f_{i}\left(s_{i}\right) f_{j}\left(s_{j}\right)\right]$ in Eqs. $(10)$ and $(23)$ becomes $\left[f_{i}(s) f_{j}(0)\right]$ which is similar to the corresponding quantity

$$
\left[f_{i}(s) f_{j}(0)+f_{i}(0) f_{j}(s)\right] / 2
$$

occurring in the leading term expressions of Bonham and Peixoto, and Yates. It is identical if atoms $i$ and $j$ are the same kind of atom.

Another type of scattering considered by Bonham and Peixoto ${ }^{5}$ and by Yates and Tenney ${ }^{8,18}$ is the case where atoms $k$ and $i$ are the same atom. This term is less interesting to structural chemists than the $i \neq j \neq k$ terms, for it is virtually devoid of structural detail. If $\boldsymbol{r}_{k}$ is set equal to zero in Eqs. (10) and (23) (naturally leading to $s_{i}=s, s_{j}=0$ ), these equations give the same result as the previously published expressions.

An identification of the role of the new corrections in terms of the discussion of Sec. $I I$ is as follows. The modulation $\exp \left(i \mathrm{~s} \cdot \mathrm{K}_{0} z_{j k}\right)$ of Eq. (12) and subsequent equations simply represents the interference resulting from the $z$ component of the separation of the $j k$ pair of scatterers. The complex Gaussian modulation $\exp \left[-\left(i r_{i j} / 2 k\right) s_{i}^{2}\right]$ of Eq. (16) expresses the effect of delocalization of the perpendicular component $p_{j k}$ of the $j k$ interference distance as weighted by the complex Gaussian shadow amplitude function $\exp \left[-\left(k / 2 i r_{i}\right) b_{i}^{2}\right]$ experienced by atom $j$ in the wake of atom $i$. This modulation is formally equivalent to that of the real Gaussian damping function $\exp \left[-\left(l^{2} / 2\right) s^{2}\right]$ included in conventional diffraction equations to account for the delocalization of an internuclear distance over a Gaussian distribution function $\exp \left[-\left(1 / 2 l^{2}\right)(\Delta r)^{2}\right]$.

The effect of the present modification is quite small if atom $k$ has a $z$-coordinate close to that of atoms $i$ or $j$ but drastic if atom $k$ is far removed. This behavior is illustrated in Fig. 2 for a series of three-atom fragments struck by $40 \mathrm{kV}$ electrons. In the computation of curves for Fig. 2, the scattering factors used were not Glauber $f_{i}(s)$ but were partial wave factors published by Schafer, Yates, and Bonham. ${ }^{19}$ A comparison of Figs. 2(b) and 2(c) reveals that the Glauber curves 2(b) begin to differ significantly from the shadow propagation curves beyond the Glauber limit of $\mathrm{Eq}$. (1b) with $d=(n+1) a_{0} / \sqrt{2}$, except for the $90^{\circ}$ bond angle special case $(n=0)$. Presumably, then, the new expressions will appreciably modify the dynamic corrections calculated for very small, randomly oriented crystallites. The virtue of the present theory is that it can be applied to tiny clusters of atoms with arbitrary, irregular sur faces, and with any desired structure defects. It will be 
illuminating to discover whether the present theory, extended to its upper limit of validity in crystallite size (that for which single-double scatterings become a marginally adequate account of the total multiple scattering) interfaces with the Blackman-Fujimoto theory ${ }^{20,21}$ extrapolated downwards from large, regular arrays of atoms.

\section{ACKNOWLEDGMENTS}

The stimulation to carry out this work was provided by calculations by and discussions with Dr. B. Raoult, Dr. G. Torchet, and Dr. J. Farges. Numerical calculations to test the new expressions were carried out by Mr. D. A. Rosenthal.

\footnotetext{
* Research supported by a grant from the National Science Foundation.

${ }^{1}$ P. J. Bunyan, Proc. Phys. Soc. London 82, 1051 (1963).

${ }^{2}$ J. Gjónnes, Acta Cryst. 13, 1075 (1964).

${ }^{3}$ R. A. Bonham, J. Chem. Phys. 43, 1103 (1965).

${ }^{1}$ R. A. Bonham, Trans. Am. Cryst. Assoc. 2, 165 (1966).

${ }^{5}$ R. A. Bonham and E. M. A. Peixoto, J. Chem. Phys, 56, 2377 (1972).
}

${ }^{6}$ J. W. Liu and R. A. Bonham, J. Mol. Struct. 11, 297 (1972).

${ }^{7}$ L. S. Bartell and T. C. Wong, J. Chem. Phys, 56, 2364 (1972).

${ }^{8}$ A. C. Yates, J. Chem. Phys. 57, 1636 (1972).

${ }^{9}$ T. C. Wong and L. S. Bartell, J. Chem. Phys. 58, 5654 (1973).

${ }^{10}$ V. C. Romanov, A. V. Neymark, M. G. Anashkin, Zhur. Strukt. Khim. 15, 383 (1974).

${ }^{11}$ E. J. Jacob and L. S. Bartell, J. Chem. Phys. 53, 2231 (1970).

${ }^{12}$ E. J. Jacob, H. B. Thompson, and L. S. Bartell, J. Mol. Struct. 8, 383 (1971).

${ }^{13}$ C. L. Ritz, Doctoral dissertation, The University of Michigan, 1975.

${ }^{14}$ A. H. Karrenbrock, Doctoral dissertation, The University of Michigan, 1975.

${ }^{15}$ B. Raoult, G. Torchet, J. Farges, and L. S. Bartell (unpublished research).

${ }^{16}$ (a) R. J. Glauber, Lect. Theor. Phys, 1, 315 (1959). (b) V. Franco and R. J. Glauber, Phys. Rev. 142, 1195 (1966).

${ }^{17} \mathrm{Or}$, in a modified treatment extending the angular range somewhat, the "thin film" is perpendicular to $\left(\mathbf{k}_{\mathbf{4 n e}}+\mathbf{k}_{\text {eeat }}\right)$.

${ }^{18}$ A. C. Yates and A. Tenney, Phys. Rev. A 5, 2474 (1972).

${ }^{19}$ L. Schafer, A. C. Yates, and R. A. Bonham, J. Chem. Phys, 55, 3055 (1971).

${ }^{20}$ M. Blackman, Proc. Roy. Soc. London 173, 68 (1939).

${ }^{21}$ F. Fujimoto, Z. Naturforsch, 20a, 367 (1965). 